\title{
Механизм и закономерность снижения светового потока светодиодов на основе структур AIGaN/InGaN/GaN с квантовыми ямами при длительном протекании прямого тока различной плотности
}

\author{
(С) Ф.И. Маняхин \\ Национальный исследовательский технологический университет „МИСиС“, \\ 119049 Москва, Россия \\ E-mail: zaomisis@yandex.ru
}

(Получена 24 мая 2017 г. Принята к печати 25 мая 2017 г.)

Установлен механизм снижения светового потока светодиодов на основе гетеростуктур AlGaN/InGaN/ $\mathrm{GaN}$ с квантовыми ямами. Уменьшение светового потока связано с генерацией точечных дефектов в активной области гетероструктур вследствие взаимодействия кристаллической решетки полупроводника с горячими носителями заряда, образующимися в режиме отклонения вольт-амперной зависимости от экспоненты. Получено аналитическое выражение закономерности спада светового потока в процессе длительного протекания тока, которое подтверждается экспериментальными результатами. Показано, что на ход зависимости светового потока от времени работы в значительной степени влияет неоднородность распределения индия в квантовых ямах.

DOI: 10.21883/FTP.2018.03.45625.8341

\section{1. Введение}

Деградация светового потока светодиодов является проблемой с начала их промышленного производства и применения. Для светодиодов и излучателей различных составов соединений и технологий в свое время были определены причины изменения их параметров во времени [1], была усовершенствована их технология.

Но с появлением новых, сверхъярких светодиодов на основе нитрида галлия с квантовыми ямами проблема деградации сохранилась. Исследованию этого явления посвящено достаточно большое количество работ [2-10]. В [11] говорится, что срок службы современных светодиодов на основе гетероструктур $\mathrm{AlGaN} / \mathrm{InGaN} / \mathrm{GaN}$ с квантовыми ямами больше 50000 ч. Кроме того, нет четкого критерия для времени наработки: при каком уровне уменьшения светового потока время будет считаться временем работы светодиода.

Нет единого мнения о причинах и механизмах спада светового потока, его закономерности во времени. Недостаточно подробно по интервалам времени строятся деградационные зависимости, что приводит к потере важной информации по спаду светового потока.

Исследованию указанных пробелов посвящена настоящая работа, целью которой является детальное изучение закономерности спада светового потока светодиодов белого свечения во времени в различных условиях эксплуатации, выявлению механизма гашения люминесценции и причин, способствующих этому.

\section{2. Постановка эксперимента, экспериментальные методики и результаты}

Исследованию подвергались серийные светодиоды белого свечения фирмы Cree на основе кристаллов с гете- роструктурой $\mathrm{AlGaN} / \mathrm{InGaN} / \mathrm{GaN}$ с квантовыми ямами C503D-WAN-CCBD231, для которых рабочий ток 20 мA, напряжение прямого смещения $3.2 \mathrm{~B}$.

Через них пропускали прямые стабилизированные токи 3, 20, 50 и $80 \mathrm{MA.} \mathrm{Световой} \mathrm{поток} \mathrm{измеряли}$ с помощью прибора ТКА-КК1 с интегрирующей сферой Ульбрихта. Диаметр измерительного шара 180 мм. Минимальная цена цифровой шкалы измерительного блока 0.01 лм.

Строились зависимости величины светового потока $L$ от времени наработки (рис. 1 ), рассчитывалась производная изменения светового потока по времени $\Delta \ln (L) / \Delta t$ (рис. 2-4).

\section{3. Обсуждение экспериментальных результатов}

Как видно из экспериментальных распределений (рис. 1), спад светового потока в полулогарифмических координатах $\ln (L(t))=f(t)$ происходит нелинейно. На этих зависимостях можно выделить три характерных экспоненциальных участка. Производная по времени для них (рис. 2-4), имеет характерную особенность: она изменяется от некоторой начальной величины, проходит через минимум, а затем увеличивается, стремясь к первоначальному значению. Нужно отметить, что характер поведения производной сохраняется для всех значений прямого тока, при которых осуществлялась наработка. Испытания светодиода зеленого свечения той же фирмы при 70 мА показали те же характерные результаты.

Для объяснения полученных результатов начнем с анализа вольт-амперных зависимостей исследуемых светодиодов. Как не раз было отмечено в предыдущих работах $[12,13]$, если не учитывать туннельную составляющую, которая проявляется при токах значительно ниже 


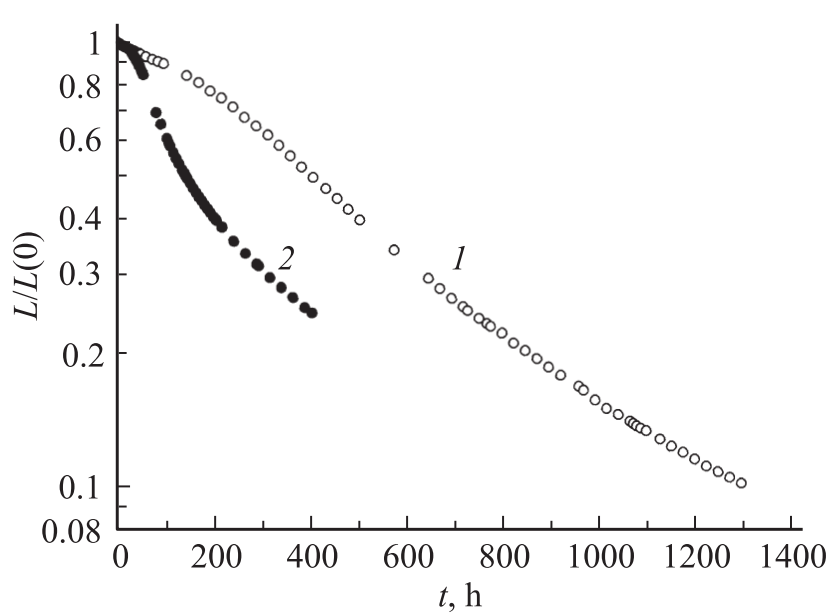

Pис. 1. Зависимость относительного светового потока от времени протекания прямого тока 20 (1) и 50 (2) мА.

рабочих, на ВАХ в полулогарифмических координатах можно выделить два основных участка:

1) экспоненциальный, соответствующий зависимости

$$
I=I_{0} \exp \left(U_{b} / m k T\right)
$$

где $I-$ прямой ток через диод, $I_{0}-$ ток насыщения, $U_{b}$ - напряжение прямого смещения, $m-$ фактор неидеальности, $k$ - постоянная Больцмана, $T-$ абсолютная температура $p-n$-перехода.

2) сублинейный участок, т.е. участок отклонения от экспоненциальной зависимости ВАХ.

В ряде работ $[14,15]$ это отклонение объясняется наличием в цепи $p-n$-перехода некоего последовательного сопротивления $R$ протеканию прямого тока, вследствие чего ход BAX на этом участке описывается выражением:

$$
I=I_{0} \exp \left(\frac{U_{b}+I R}{m k T}\right) .
$$

Анализ ВАХ на этом участке свидетельствует о том, что это сопротивление зависит от прямого тока и не может быть интерпретировано как постоянный резистор.

В связи с этим логично предположить, что внутри ОПЗ образуется участок, на котором возникает дрейфовое электрическое поле. На этом участке падает часть прямого напряжения, которое обозначим $U_{i}$. Тогда выражение (2) примет вид

$$
I=I_{0} \exp \left(\frac{U_{b}+U_{i}}{m k T}\right)
$$

Возникает вопрос - какова же природа этого напряжения? Учет распределения заряда в ОПЗ широкозонных полупроводников позволяет дать ответ на этот вопрос.

В работе [12] было показано, что светодиодную структуру на основе $\mathrm{GaN}$ нужно представлять в виде $p-i-n$-структуры с $i$-слоем шириной порядка $10^{-6} \mathrm{~cm}$,

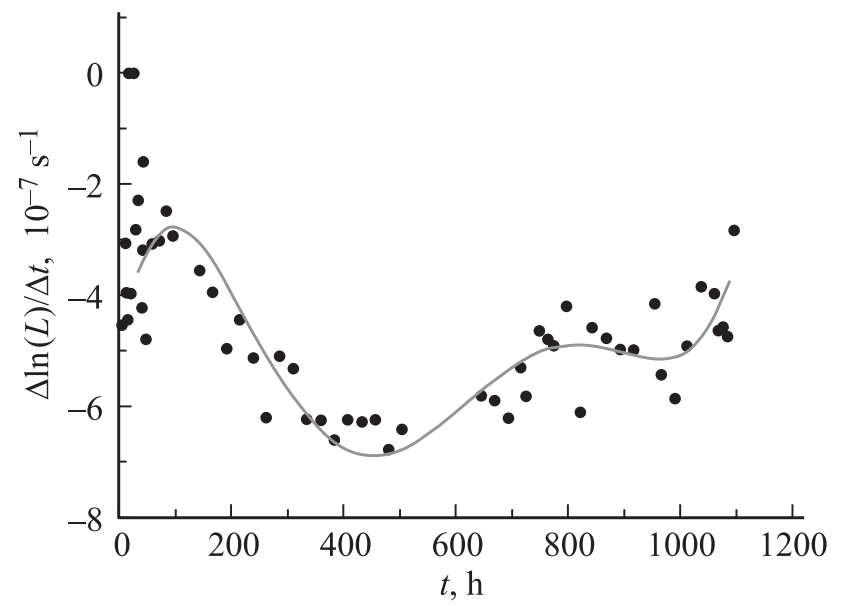

Рис. 2. Зависимость $\Delta \ln (L) / \Delta t$ от времени наработки при прямом токе $20 \mathrm{мA}$; сплошная линия - полином пятой степени для распределения массива экспериментальных точек.

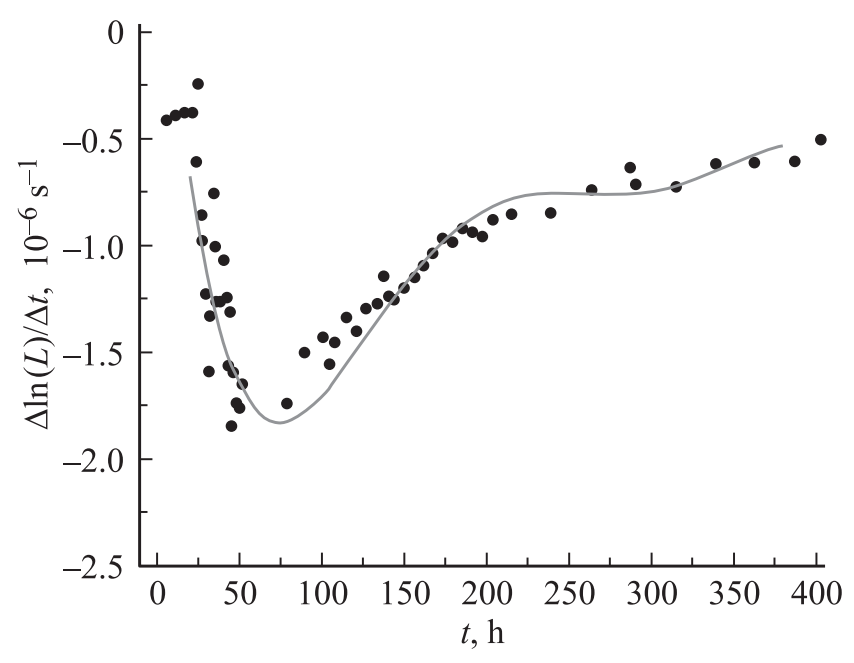

Рис. 3. Зависимость $\Delta \ln (L) / \Delta t$ от времени наработки при прямом токе 50 мА; сплошная линия - полином пятой степени для распределения массива экспериментальных точек.

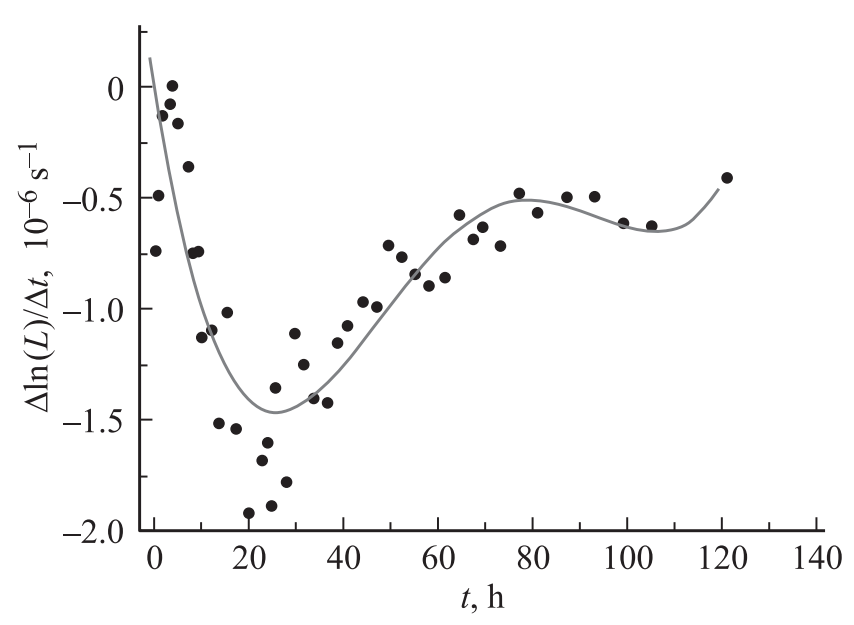

Рис. 4. Зависимость $\Delta \ln (L) / \Delta t$ от времени наработки при прямом токе $70 \mathrm{MA}$; сплошная линия - полином пятой степени для распределения массива экспериментальных точек. 
и в отличие от резкого $p-n$-перехода, в котором распределение электрического поля имеет треугольный вид, электрическое поле в $i$-слое будет определяться наличием в нем свободных носителей заряда.

Анализ распределения электрического поля на границе легированного и компенсированного слоев будем проводить без учета квантовых ям.

Чтобы понять суть обсуждаемого явления, рассмотрим в качестве примера энергетическую структуру контакта $n$ и $n_{i}^{+}$слоев. Здесь $n_{i}^{+}$обозначена концентрация заряда компенсированного слоя (КС) с преобладанием доноров. Электрическое поле на границе легированного слоя и КС рассчитывается из условия, что концентрация диффундировавших в КС электронов много больше концентрации неподвижных скомпенсированных ионов примеси. Электроны из сильно легированного слоя диффундируют в КС, оставляя в нем положительно заряженные ионы донорной примеси. Между слоями подвижных электронов и ионов создается электрическое поле $\varepsilon$, которое дрейфовым потоком уравновешивает диффузионный поток. В стационарном состоянии при прямом напряжении смещения для плотности тока через контакт $n-n_{i}^{+}$будет справедливо равенство

$$
q \Delta n \mu_{n} \varepsilon-q D_{n} \frac{d \Delta n}{d x}=J_{n \text { dif }}
$$

где $J_{n \text { dif }}$ - диффузионный ток от $n$ слоя в глубь КС.

При напряжении смещения $U_{b}=0 J_{\text {ndif }}=0$ и дрейфовый и диффузионный потоки равны. При приложении прямого напряжения смещения диффузионный поток становится меньше дрейфового вследствие уменьшения градиента концентрации электронов. Длина экранирования электронов увеличивается и электроны в большей концентрации проникают в глубь КС.

Отношение дрейфового потока к диффузионному в компенсированном слое можно записать отношением

$$
\frac{\Delta n \mu_{n} \mathcal{\varepsilon}}{D_{n} \frac{d \Delta n}{d x}}=(1+\beta),
$$

где $\beta$ может изменяться от 0 до $\infty$ в зависимости от величины напряжения смещения.

Для слоя электронов в КС можно записать уравнение

$$
\varepsilon=\frac{(1+\beta) D_{n}}{\mu_{n}\left(n_{i}^{+}+\Delta n\right)} \frac{d \Delta n}{d x}
$$

где $D_{n}, \mu n-$ коэффициент диффузии и подвижность электронов соответственно, $\Delta n-$ избыточная концентрация продиффундировавших в КС электронов.

Учитывая, что при $D_{n}=\frac{k T}{q} \mu$, при $\Delta n \gg n_{i}^{+}$

$$
\varepsilon=\frac{(1+\beta) k T}{q \Delta n} \frac{d \Delta n}{d x}
$$

Продифференцировав (7), получаем выражение для плотности подвижного заряда в КС:

$$
\begin{aligned}
\frac{d \varepsilon}{d x} & =\frac{(1+\beta) k T}{q} \frac{d}{d x}\left(\frac{1}{\Delta n} \frac{d \Delta n}{d x}\right) \\
& =\frac{(1+\beta) k T}{q}\left[-\frac{1}{\Delta n^{2}}\left(\frac{d \Delta n}{d x}\right)^{2}+\frac{1}{\Delta n} \frac{d^{2} \Delta n}{d x^{2}}\right]
\end{aligned}
$$

В другом виде плотность заряда в КС можно записать выражением

$$
\frac{d \varepsilon}{d x}=-\frac{q}{\varepsilon \varepsilon_{0}} \Delta n
$$

где $\varepsilon, \varepsilon_{0}-$ относительная и абсолютная диэлектрические проницаемости полупроводника.

Приравняв (8) и (9), получим

$$
\frac{d \Delta n^{2}}{d x^{2}}=\left[\frac{1}{\Delta n}\left(\frac{d \Delta n}{d x}\right)^{2}-\frac{q^{2}}{(1+\beta) k T \varepsilon \varepsilon_{0}}(\Delta n)^{2}\right] .
$$

В характеристическом виде (10) примет вид

$$
y^{\prime \prime}=\left[\frac{\left(y^{\prime}\right)^{2}}{y}-\frac{b y^{2}}{(1+\beta)}\right] .
$$

В нем обозначены: $y$ - функция распределения свободных носителей заряда в КС, $b=\frac{q^{2}}{k T \varepsilon \varepsilon_{0}}$.

Подобные рассуждения справедливы и для контакта $p-n_{i}^{+}$.

Таким образом, в КС образуются заряженные слои подвижных носителей заряда, электрическое поле которых направлено против электрического поля неподвижных ионов примеси легированных слоев.

Численным моделированием с применением пакета MATHCAD были получены распределения свободных носителей заряда, электрического поля и потенциала в ОП3 $p-n_{i}^{+}-n$-перехода без квантовых ям.

При моделировании были заданы следующие параметры $p-n_{i}^{+}-n$-перехода: $N_{d}=1.5 \cdot 10^{19} \mathrm{~cm}^{-3}, N_{a}=$ $=3.0 \cdot 10^{18} \mathrm{~cm}^{-3}$, ширина КС $-X_{0}=3.2 \cdot 10^{-6}$ см. Эти параметры взяты из экспериментальных измерений распределения концентрации зарядовых центров [12].

Диаграммы показывают, что образующийся слой диффундирующих электронов со стороны $n$-слоя и дырок со стороны $p$-слоя экранируют электрическое поле ионов $n$ - и $p$-областей. Вследствие этого в компенсированном слое, в отличие от классического линейного распределения поля области $p-n$-контактов, поле КС изменяется с переходом от границы $n-n_{i}^{+}$к границе $n_{i}^{+}=p$, проходя минимум. Этот минимум становится более выраженным при увеличении напряжения прямого смещения.

При некотором напряжении прямого смещения электрическое поле КС становится равным нулю, а при дальнейшем увеличении напряжения смещения оно меняет знак на противоположный и становится дрейфовым для инжектированных носителей заряда, преодолевших барьеры со стороны ОПЗ ионов легированных слоев. 
Результаты моделирования распределения электрического поля КС показывают, что его величина имеет порядок $10^{5} \mathrm{~B} / \mathrm{cm}$, что создает условия насышения дрейфовой скорости и возникновение горячих носителей заряда (рис. 5,a). В результате падение прямого напряжения на диоде складывается из напряжения, идущего на снижение потенциальных барьеров в $n$ - и $p$-слоях $U_{b}$, и напряжения области дрейфового поля $U_{i}$ (рис. $\left.5, b\right)$.

Логично предположить, что в КС нарушается режим термодинамического равновесия: горячие носители заряда и кристаллическая решетка представляют собой две системы, обменивающиеся энергией, отличной от $k T_{0}$, где $T_{0}-$ температура решетки. Вследствие передачи энергии горячими носителями заряда атомам решетки образуются неравновесные точечные дефекты, которые гасят люминесценцию.

Пусть горячие электроны в КС имеют температуру $T_{e}$, в то время как температура самой решетки $T_{0}<T_{e}$.

Баланс скоростей изменения свободной энергии образования избыточной концентрации точечных дефектов $E_{d} \Delta N_{d}$ и энергий кристаллической решетки $\Delta S_{A}$, а также системы горячих электронов $S_{e}$ в установившемся режиме будет определяться соотношением:

$$
\begin{gathered}
\frac{\partial^{2} \Delta G}{\left(\partial \Delta N_{d}\right)^{2}}=\frac{\partial^{2}}{\left(\partial \Delta N_{d}\right)^{2}}\left[T_{e} S_{e}-\left(\frac{E_{d}}{2} \Delta N_{d}-T_{0} \Delta S_{A}\right)\right] \\
=\frac{\partial}{\left(\partial \Delta N_{d}\right)^{2}}\left\{k T_{e} \ln \left[\frac{\left(n_{e}+\frac{\Delta N_{d}}{\tau_{A}}\right) !}{n_{e}\left(\frac{\Delta N_{d}}{\tau_{A}}\right) !}\right]\right. \\
\left.-\frac{\frac{E_{d}}{2} \Delta N_{d}-k T_{0} \ln \left[\frac{\left(N_{0}+\Delta N_{d}+\Delta-N_{C}\right) !}{N_{0} !\left(\Delta N_{d}+\Delta N_{C}\right) !}\right]}{\tau_{A}}\right\}=0,
\end{gathered}
$$

где $\Delta N_{d}, \Delta N_{C}-$ неравновесная и равновесная концентрации дефектов по Френкелю (для конкретности имеются в виду электрически неактивные точечные дефекты), $N_{0}$ - концентрация атомов кристаллической решетки, $E_{d}-$ необходимая минимальная энергия для смещения атома в междоузлие; так как в результате смещении образуется вакансия, $E_{d}$ делится пополам, $\tau_{A}-$ среднее время существования неравновесных точечных дефектов в режиме рекомбинационно-стимулированной диффузии, $n_{e}$ - частота столкновений горячих электронов с атомами решетки с релаксацией по энергии. Изменение энтропии горячих электронов связано с образованием изолированных дефектов: потеря энергии электронами приводит к увеличению их конфигурационной энергии и энтропии кристаллической решетки вследствие смещения атома из узла.

С учетом того, что деградационные эффекты проявляются при $\Delta N_{d} \gg \Delta N_{C}$ и что $n_{e} \tau_{A} \gg N_{d}$, из соотношения (12) получим

$$
\frac{E_{d}}{2}+k T_{e} \ln \frac{\Delta N_{d}}{N_{0}}+k T_{e} \ln \frac{\Delta N_{d}}{n_{e} \tau_{A}}=0 .
$$

Число электронов, прошедших через единицу площади гетероперехода в единицу времени, равно $J / q$. Если
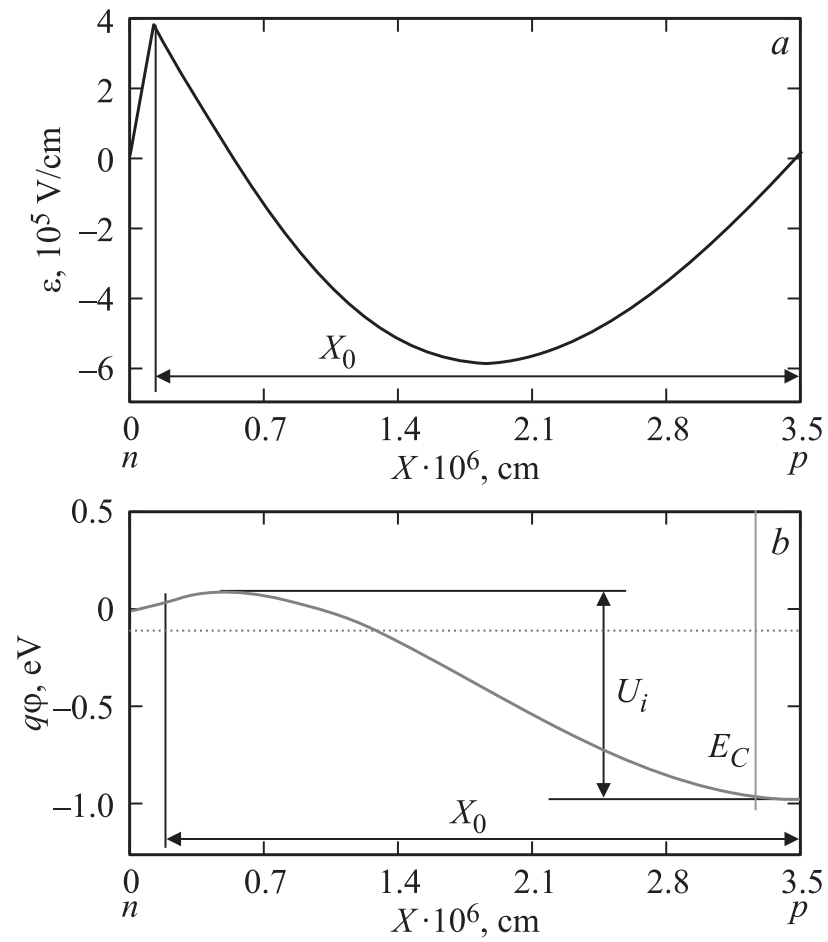

Pис. 5. Диаграммы электрического поля $(a)$ и потенциала дна зоны проводимости $(b)$ в ОПЗ $p-n_{i}^{+}-n$-перехода на участке BAX неэкспоненциальной зависимости; $X_{0}$ - ширина компенсированного слоя; $n, p-$ обозначение легированных областей $p-n_{i}^{+}-n$-перехода.

принять, что средняя длина свободного пробега электронов с передачей всей кинетической энергии равна $l_{e}=\left(\sigma N_{0}\right)^{-1}$, где $\sigma_{e}-$ сечение взаимодействия при релаксации по энергии, то $n_{e}=\frac{3 J \sigma_{e} N_{0}}{2 q}$, а выражение для скорости генерации дефектов примет вид

$$
G_{d}=\frac{3 N_{0}}{2} \frac{J}{q} \sigma_{e} \exp \left(-\frac{E_{d}}{2(k T+\Delta E)}\right)
$$

где $\Delta E-$ энергия горячих электронов.

При насыщении дрейфовой скорости в материалах $A^{\mathrm{III}} B^{\mathrm{V}}$ рассеяние происходит в основном на оптических фононах $\hbar \omega_{0}$, энергия которых практически не зависит от волнового вектора. В большинстве литературных источников утверждается, что в этом случае дрейфовая скорость выражается формулой

$$
V_{\mathrm{dr} . \mathrm{Sat}}=\left(\frac{\hbar \omega_{0}}{m^{*}}\right)^{0.5}
$$

где $m^{*}$ - эффективная масса носителей заряда, $V_{\mathrm{dr.Sat}}$ дрейфовая скорость в насыщении.

Однако надо учитывать, что в общем дрейфовом потоке участвуют горячие носители заряда, имеющие энергию в электрическом поле много больше $\hbar \omega_{0}$, и они теряют эту энергию при многофононном рассеянии. Полный поток горячих носителей складывается из парциальных потоков с энергией $n \hbar \omega_{0}$, где $n=1,2,3 \ldots N$. 
Поток частиц $U_{\mathrm{drn}}$ с энергией $n \hbar \omega_{0}$ и концентрацией $N_{n}$ можно оценить по формуле

$$
U_{\mathrm{drn}}=\left(\frac{n \hbar \omega_{0}}{m^{*}}\right)^{0.5} N_{n} .
$$

Если принять, что в стационарном неравновесном режиме вероятности образования и поглощения горячими носителями заряда одного неравновесного фонона $\hbar \omega_{0}$ одинаковы $(p=0.5)$, то среднюю дрейфовую скорость при многофононной передаче энергии горячими носителями заряда можно выразить уравнением

$$
V_{\mathrm{drSat}}=\frac{1}{N_{e}} \sum_{n=1}^{\infty}\left[\left(\frac{n \hbar \omega_{0}}{m^{*}}\right)^{0.5} N_{n}\right]=\sum_{n=1}^{\infty}\left(\frac{n \hbar \omega_{0}}{n^{*}}\right)^{0.5} p^{n},
$$

где $N_{e}$ - общая концентрация частиц потока, $p^{n}-$ вероятность многофононной передачи энергии кристаллической решетке горячими носителями заряда.

Для стационарного режима события возникновения или исчезновения одного фонона противоположны и образуют полную группу. Сумма их вероятностей равна 1, а для того чтобы процесс образования фонона и его исчезновения был стационарным, вероятности этих событий должны быть равными. Средняя энергия потока носителей заряда при многофононном рассеянии определяется выражением

$$
\Delta E=\left[\sum_{n=1}^{\infty}\left(n \hbar \omega_{0}\right)^{0.5} p^{n}\right]^{2}
$$

K примеру, для $\mathrm{GaN} \hbar \omega_{0}=0.091$ эВ, в этом случае $\Delta E=0.165$ эВ. Таким образом, в режиме насыщения дрейфовой скорости в электрическом поле КС наиболее вероятным является двухфононное рассеяние. При этом для согласия со справочной величиной $V_{\mathrm{drSat}}$ эффективная масса электрона должна составлять $m^{*}=0.35 m_{0}$. По справочным данным, $m^{*}=0.2 m_{0}$ и значение средней расчетной массы может быть объяснено эффектом перехода электронов в боковую долину с большей эффективной массой.

В режиме насыщения дрейфовой скорости среднее время свободного пробега $\tau_{\text {fre }}$ при рассеянии можно рассчитать по формуле

$$
\tau_{\mathrm{fre}}=\frac{1}{N_{q} \sigma_{q} V_{\mathrm{drSat}}}
$$

где $N_{q}=1 / l_{\mathrm{ave}}^{3}-$ концентрация центров рассеяния, $l_{\text {ave }}-$ средняя длина свободного пробега, $\sigma_{q}=l_{\text {ave }}^{2}-$ ceчение рассеяния. В результате получим $\tau_{\mathrm{fre}}=l_{\mathrm{ave}} / V_{\mathrm{drave}}$, где $V_{\mathrm{dr}, \mathrm{ave}}-$ средняя дрейфовая скорость потока электронов с различной энергией.

Выразим среднюю длину свободного пробега формулой

$$
l_{\mathrm{ave}}=\frac{m^{*} V_{\mathrm{drave}}^{2}}{q E}=\frac{m^{*} V_{\mathrm{drave}}^{2} X}{q U_{i}},
$$

где $U_{i}$ - падение напряжения на компенсированном слое, $X$ - ширина компенсированного слоя, где создается сильное электрическое поле. Нужно отметить, что
Таблица 1. Коэффициенты $k_{L}$, полученные расчетным путем и из экспериментальных зависимостей на начальном участке деградации при различных прямых токах

\begin{tabular}{c|c|c|c}
\hline \multirow{2}{*}{ Образец } & \multirow{2}{*}{$\begin{array}{c}\text { Прямой ток, } \\
\text { мА }\end{array}$} & \multicolumn{2}{|c}{ Коэффициент $k_{L}, \mathrm{c}^{-1}$} \\
\cline { 3 - 4 } & & экспериментальный & расчетный \\
\hline $0 w$ (белый) & 20 & $2.9 \cdot 10^{-7}$ & $3.0 \cdot 10^{-7}$ \\
$1 w$ (белый) & 50 & $2.0 \cdot 10^{-6}$ & $2.0 \cdot 10^{-6}$ \\
$8 w$ (белый) & 80 & $7.0 \cdot 10^{-6}$ & $5.0 \cdot 10^{-6}$ \\
$2 w$ (белый) & 3 & $2.6 \cdot 10^{-8}$ & $1.3 \cdot 10^{-8}$ \\
$3 w$ (белый) & 1 & $1.95 \cdot 10^{-9}$ & $2.1 \cdot 10^{-9}$
\end{tabular}

ширина КС изменяется от 0 до $X_{0}$ с момента отклонения BAX от экспоненциальной зависимости.

Используя выражение (14), с учетом сделанных замен для скорости генерации точечных дефектов получим окончательно выражение

$$
G_{d}=\frac{3}{2} \frac{J U_{i}}{X_{0} \Delta E} e^{\frac{-E_{d}}{2(k T+\Delta E)}} .
$$

Полученное выражение позволяет дать ответ на особенности экспериментальных зависимостей спада светового потока при длительном протекании прямого тока различной плотности.

В общем случае дифференциальное уравнение изменения светового потока можно записать в виде

$$
\frac{d L}{d t}=-k_{L} L
$$

где $L-$ световой поток, $k_{L}-$ коэффициент с размерностью $1 / \mathrm{c}$.

Если принять, что концентрация центров излучения равна $N_{L}$, а концентрация атомов решетки $N_{0}$, то скорость убывания центров люминесценции будет пропорциональна отношению $N_{L} / N_{0}$ и $G_{d}$, т.е.

$$
k_{L}=\frac{G_{d}}{N_{0}}
$$

Так как световой поток пропорционален концентрации излучательных центров, то выражение для его зависимости от времени примет вид

$$
L(t)=L_{0} \exp \left(-k_{L} t\right)
$$

Коэффициенты $k_{L}$, полученные из экспериментальных зависимостей $L(t)$ на начальных участках деградации и рассчитанные по (23), приведены в табл. 1. В выражении (21) $E_{d}=9$ эВ [14], $X_{0}=1 \cdot 10^{-6} \mathrm{cм}, \Delta E=0.165$ эВ, в выражении (23) $N_{0}=2.5 \cdot 10^{23} \mathrm{~cm}^{-3}$.

Стоит согласиться с достаточно хорошим совпадением экспериментальных и расчетных параметров.

Однако тут же встает закономерный вопрос - какова причина изменения $k_{L}$ при дальнейшем увеличении времени наработки. Как следует из (14), этого изменения не должно быть: все параметры этого выражения 
Таблица 2. Распределение индия по слою квантовой ямы в различные периоды наработки светодиода при сохранении интегральной плотности тока

\begin{tabular}{c|c|c|c|c}
\hline $\begin{array}{c}\text { Содержание } \\
\text { в различных } \\
\text { областях квантовой } \\
\text { ямы (Кя), } x\end{array}$ & $\begin{array}{c}\text { Начальное } \\
\text { распределение } \\
\text { In по слою КЯ } \\
{[16]}\end{array}$ & $\begin{array}{c}\text { Распределение In } \\
\text { по слою КЯ в период } \\
\text { изменения } k_{L} \\
\text { (гипотетическое) }\end{array}$ & $\begin{array}{c}\text { Распределение In } \\
\text { после длительной } \\
\text { наработки } \\
\text { (гипотетическое) }\end{array}$ & $\begin{array}{c}\text { Плотность тока } \\
\text { через парциальные } \\
\text { диоды слоя КЯ } \\
{[16]}\end{array}$ \\
\hline 0.14 & 0 & 0 & 0 & 485.7 \\
0.15 & 0.005 & 0 & 0 & 270.6 \\
0.16 & 0.01 & 0.05 & 0 & 149.8 \\
0.17 & 0.05 & 0.2 & 0.05 & 54.4 \\
0.18 & 0.12 & 0.5 & 0.2 & 31.6 \\
0.20 & 0.24 & 0.2 & 0.2 & 23.8 \\
0.21 & 0.20 & 0.183 & 0.05 & 24.1 \\
0.23 & 0.12 & 0.05 & 0 & 31.3 \\
0.24 & 0.05 & 0.03 & 0 & 42.5 \\
\end{tabular}

практически постоянны - задан ток наработки, ширина компенсированного слоя, напряжение $U_{i}$ практически постоянно, так же как и энергия горячих электронов $\Delta E$ и энергия смещения $E_{d}$.

На этот вопрос помогут ответить материалы работ $[15,16]$.

В работе [16] кристалл светодиода модельно представлен в виде параллельно включенных диодов с различной шириной квантовых ям, обусловленной флуктуацией содержания индия по площади квантовой ямы светодиода. Плотность тока через парциальные диоды с малым содержания индия $x$ (например, при $x=0.15$ ) может быть в несколько раз больше, чем для диодов с $x=0.25$. В [16] принято среднее значение $x=0.2$ (для светодиода синего свечения) с нормальным распределением, представленным в табл. 2.

В процессе наработки наиболее интенсивная генерация точечных дефектов происходит именно в областях с меньшим содержанием индия. Через определенное время концентрация вакансий становится достаточной для диффузии индия по слою квантовой ямы и происходит его перераспределение со смещением его содержания в области меньшего $x$ (табл. 2). При этом увеличивается парциальная плотность прямого тока по областям с меньшим содержанием $x$ при сохранении средней плотности по кристаллу. Увеличение экспозиции наработки увеличивает концентрацию вакансий кристаллической решетки, которая способствует выравниванию индия по слою квантовой ямы. Среднее его значение сохраняется, а распределение по слою стремится к малому значению среднеквадратического отклонения (табл. 2). На графике зависимости светового потока от времени наблюдается уменьшение абсолютного значения $k_{L}$, близкого к начальному.

В табл. 3 приведены расчетные значения $k_{L}$ при плотности тока $20 \mathrm{~A} / \mathrm{cm}^{2}$ для распределений табл. 2
Таблица 3. Расчетные коэффициенты $k_{L}$ для распределений табл. 2

\begin{tabular}{l|r}
\hline \multicolumn{1}{c|}{ Распределение } & \multicolumn{1}{c}{$k_{L}, \mathrm{c}^{-1}$} \\
\hline Начальное распределение & $1.25 \cdot 10^{-7}$ \\
Распределение при средней экспозиции & $2.4 \cdot 10^{-7}$ \\
Распределение при большой экспозиции & $9.4 \cdot 10^{-8}$
\end{tabular}

без учета локального перегрева областей с высокой плотностью тока.

Таким образом, сделанный анализ причин непостоянства $k_{L}$ согласуется с экспериментальными результатами, представленными в настоящей работе и согласуется с выводами приведенных публикаций.

\section{4. Заключение}

На основании материалов представленной работы можно сделать следующие выводы.

1. Одной из основных причин спада светового потока светодиодов белого свечения на основе гетероструктур $\mathrm{AlGaN} / \mathrm{InGaN} / \mathrm{GaN}$ с квантовыми ямами является образование точечных дефектов из-за воздействия горячих электронов $p-i-n$-перехода на кристаллическую решетку полупроводника в режиме отклонения ВАХ от экспоненциальной зависимости.

2. Причиной возникновения горячих электронов в компенсированном слое в режиме отклонения ВАХ от экспоненциальной зависимости является возникновение в нем дрейфового поля высокой напряженности от заряженных слоев свободных носителей заряда.

3. Точечные дефекты возникают в результате взаимодействия системы горячих электронов и атомов кристаллической решетки в неравновесном режиме обмена 
энергиями горючих электронов и кристаллической решетки.

4. Закономерность убывания светового потока светодиодов от времени наработки в целом подчиняется спадающей экспоненциальной зависимости с коэффициентом $k_{L}$, изменяющимся во времени вследствие перестройки неравномерного распределения индия по слоям квантовых ям.

5. Экспериментально показано, что время работы светодиодов зависит от плотности тока через них $J$ и напряжения в компенсированном слое $U_{i}$. Время спада светового потока до $50 \%$ в режиме непрерывного протекания тока не будет превышать $t=-\frac{1}{k_{L}} \ln (0.5)$. Максимальное время наработки будет для режима прямого смещения до отклонения ВАХ от экспоненциальной зависимости.

6. Светодиоды с более равномерным распределением $I_{n}$ в квантовых ямах будут обладать большим ресурсом по наработке.

Работа выполнена при финансировании в рамках программы 5-100 повышения конкурентоспособности НИТУ „МИСиС“.

\section{Список литературы}

[1] Т.В. Торчинская, Г.И. Семенова, М.К. Шейнкман. Укр. физ. журн., 34 (2),1079 (1989).

[2] Ф.И. Маняхин. Изв. вузов. Матер. электрон. техн., 2, 54 (2010).

[3] Н.И. Бочкарева, А.А. Ефремов, Ю.Т. Ребане, Р.И. Горбунов, А.В. Клочков, Ю.Т. Шретер. ФТП, 40 (1), 122 (2006).

[4] С.Г. Никифоров. Компоненты и технологии, 11, 42 (2006).

[5] К.А. Виноградова, В.Е. Бугров, А.Р. Ковш, М.А. Одноблюдов, В.И. Николаева, А.Е. Романов. Изв. вузов. Приборостроение, 56 (11), 87 (2015).

[6] И.М. Викулин, В.И. Ирха, Б.В. Коробицын, В.Э. Горбачев. Технология и конструирование в электрон. аппаратуре, $\mathbf{2}$, 55 (2004)

[7] И.В. Рожанский, Д.А. Закгейм. ФТП, 40 (2), 861 (2006).

[8] Е.К. Наими, С.Г. Никифоров, О.И. Рабинович, В.П. Сушков. Изв. вузов. Матер. электрон. техн., 1, 96 (2009).

[9] Е.К. Наими, О.И. Рабинович, В.П. Сушков. Изв. вузов. Матер. электрон. техн., 3, 58, (2006).

[10] E.K. Naimi, O.I. Rabinovich. Crystallography Reports, 56 (3), 486 (2011).

[11] Н.М. Шмидт, А.С. Усиков, Е.И. Шабувина, А.Е. Черняков, С.Ю. Курин, Ю.Н. Макаров, Х.И. Хелава, Б.П. Панченко. Науч.-техн. вестн. информационных технологий, механики и оптики, 15 (1), 46 (2015).

[12] Ф.И. Маняхин. Изв. вузов. Матер. электрон. техн., 3, 51 (2009).

[13] Ф.И. Маняхин, А.Б. Ваттана, Л.О. Мокрецова. Матер. заочной Междунар. конф. „Инновационное развитие отраслевой автоматизации, инбормационных и энергосберегающих технологий. Современное состояние, проблематика и перспективы“ // М., МИСиС, 20 декабря (2013).

[14] В.С. Вавилов, А.Е. Кив, О.Р. Ниязова. Механизмы образования и миграции дефектов в полупроводниках ( М., Наука, 1981).
[15] В.Е. Кудряшов, С.С. Мамакин, А.Н. Туркин, А.Э. Юнович, А.Н. Ковалев, Ф.И. Маняхин. ФТП, 35 (7), 861 (2001).

[16] В.П. Сушков, С.Г. Никифоров. Полупроводниковая светотехника, 3, 10, (2011).

Редактор Г.А. Оганесян

\section{The mechanism and regularity of decrease in the light stream of light-emitting diodes on the bases of structures AIGaN/InGaN/GaN with quantum wells at long courese of derect current of various density}

\section{F.I. Manyakhin}

National research technological university „MISIS“, 119049 Moscow, Russia

Abstract The mechanism of decrease in a light stream of light-emmitting diodes on the basis of heterostructures $\mathrm{AlGaN} / \mathrm{InGaN} / \mathrm{GaN}$ with quantum holes is establishe. Reduction of a light stream is connected with generation of dot defects in active area heterostructures owing to interaction of a crystal lattice of the semiconductor with the hot carriers of a charge formed in a mode of a deviation volt-ampere of dependence from exhibitors.

Analytical expression of law of recession of a light stream during long course of a current which proves to be true experimental results is received. It is shown, that the course of dependence of a light stream from an operation time substantially is influenced with heterogeneity of distribution Indea in quantum holes. 